\title{
Network-based identification of critical regulators as putative drivers of human cleft lip
}

Aimin $\mathrm{Li}^{1,2 \dagger}$, Guimin Qin ${ }^{2,3 \dagger}$, Akiko Suzuki ${ }^{4,5}$, Mona Gajera ${ }^{4,5}$, Junichi Iwata ${ }^{4,5,6}$, Peilin Jia ${ }^{2^{*}}$ and Zhongming Zhao 2, $^{*}$

From The International Conference on Intelligent Biology and Medicine (ICIBM) 2018

Los Angeles, CA, USA. 10-12 June 2018

\begin{abstract}
Background: Cleft lip (CL) is one of the most common congenital birth defects with complex etiology. While genome-wide association studies (GWAS) have made significant advances in our understanding of mutations and their related genes with potential involvement in the etiology of $\mathrm{CL}$, it remains unknown how these genes are functionally regulated and interact with each other in lip development. Currently, identifying the disease-causing genes in human $\mathrm{CL}$ is urgently needed. So far, the causative $\mathrm{CL}$ genes have been largely undiscovered, making it challenging to design experiments to validate the functional influence of the mutations identified from large genomic studies such as CL GWAS.
\end{abstract}

Results: Transcription factors (TFs) and microRNAs (miRNAs) are two important regulators in cellular system. In this study, we aimed to investigate the genetic interactions among TFs, miRNAs and the CL genes curated from the previous studies. We constructed miRNA-TF co-regulatory networks, from which the critical regulators as putative drivers in $\mathrm{CL}$ were examined. Based on the constructed networks, we identified ten critical hub genes with prior evidence in $\mathrm{CL}$. Furthermore, the analysis of partitioned regulatory modules highlighted a number of biological processes involved in the pathology of $\mathrm{CL}$, including a novel pathway "Signaling pathway regulating pluripotency of stem cells". Our subnetwork analysis pinpointed two candidate miRNAs, hsa-mir-27b and hsa-mir-497, activating the Wnt pathway that was associated with $\mathrm{CL}$. Our results were supported by an independent gene expression dataset in $\mathrm{CL}$.

Conclusions: This study represents the first regulatory network analysis of $\mathrm{CL}$ genes. Our work presents a global view of the $C L$ regulatory network and a novel approach on investigating critical miRNAs, TFs and genes via combinatory regulatory networks in craniofacial development. The top genes and miRNAs will be important candidates for future experimental validation of their functions in $\mathrm{CL}$.

Keywords: Cleft lip, Feed forward loop, Regulatory network, Disease-causing gene, microRNA, Transcription factor

\footnotetext{
*Correspondence: Peilin.Jia@uth.tmc.edu; zhongming.zhao@uth.tmc.edu

${ }^{+}$Aimin Li and Guimin Qin contributed equally to this work.

${ }^{2}$ Center for Precision Health, School of Biomedical Informatics, The University

of Texas Health Science Center at Houston, 7000 Fannin St., Suite 820,

Houston, TX 77030, USA

Full list of author information is available at the end of the article
}

(c) The Author(s). 2019 Open Access This article is distributed under the terms of the Creative Commons Attribution 4.0 International License (http://creativecommons.org/licenses/by/4.0/), which permits unrestricted use, distribution, and reproduction in any medium, provided you give appropriate credit to the original author(s) and the source, provide a link to the Creative Commons license, and indicate if changes were made. The Creative Commons Public Domain Dedication waiver (http://creativecommons.org/publicdomain/zero/1.0/) applies to the data made available in this article, unless otherwise stated. 


\section{Background}

Cleft lip (CL) is one of the most common congenital birth defects in humans, affecting approximately 1/700 live births [1]. Specifically, CL is characterized by unilateral or bilateral clefts in the upper lip resulting from failure of the growth and/or fusion of the nasal and maxillary processes during craniofacial development. CL is a multifactorial disorder, caused by a combination of genetics and environmental factors and influences feeding, speech, appearance, and more [2]. To dissect and explain complex characteristics of $\mathrm{CL}$, a deep understanding of its fundamental genetics is crucial. During the recent decade, numerous genetic studies (e.g. genome-wide association studies (GWAS), copy number variation, mRNA and miRNA expression profiling, chromatin modifier-associated region identification assay, enhancer reporter gene assay and methylation) have been performed, which substantially contributed to the discovery of human CL genes and their potential functions [3-7]. Previous studies have suggested that mutations and variations in the IRF6, p63, and TGFA genes were etiologic in CL $[1,8,9]$. Some studies indicate that $\mathrm{p} 63$ protein activates IRF6 transcription through binding its enhancer [10, 11]. p63 and IRF6 cooperate within a feedback regulatory loop in order to determine the fate of epithelial cells in proliferation vs. differentiation during palate development [12]. Disruption of this loop by mutations in either IRF6 or p63 might increase the susceptibility to CL. Although these results are still limited to unveil a systematic view of the biological process of $\mathrm{CL}$, it provides us a clue that analysis of gene regulation relationships would be powerful for identification of critical genes or regulatory motifs that drive $\mathrm{CL}$. This is also consistent with that craniofacial development is often involved in many biological processes and cascades, which can be detected at the molecular levels (e.g., gene expression, enhancer, transcription factor, and post-transcriptional regulation).

MicroRNAs (miRNAs) and transcription factors (TFs) are key regulators of gene expression. miRNAs are small non-coding RNAs (composing about 21 22 nucleotides) that regulate gene expression at the post-transcriptional level. In animals, a mature miRNA typically binds to the 3 ' untranslated regions (3'UTRs) of the target mRNAs, and consequently leads to degradation and translational repression of the mRNAs [13]. Previous studies have revealed that overexpression of miR-140 could result in CL in zebrafish [14], and that a single nucleotide polymorphism (SNP) located in pre-miR-140 was found to be associated with cleft palate (CP) in humans [15]. Recently, hundreds of miRNAs are reported to have aberrant expression in CL [16]; however, researchers have yet to find out which miRNAs play prominent roles in the pathological process of CL or the interrelated targets of these miRNAs.

Gene transcription is typically regulated by TFs in cellular systems. TFs control the rate of transcription from
DNA to mRNAs by binding to the transcription factor binding sites (TFBS) in the promoter regions of the target genes [17]. miRNAs and TFs can be co-regulated or regulated with each other by several scenarios: miRNAs' expression may be regulated by TFs [18], TFs and miRNAs may mutually regulate one another to represent feedback loops (FBLs), or alternatively, both TFs and miRNAs may simultaneously regulate their joint target genes and form feed-forward loops (FFLs). Network analysis, including motifs such as FBLs and FFLs, is an effective way to explore the fundamental global topological structures of molecular networks [19]. For example, miRNA-TF co-regulation is one of the simplest but important FFL types. So far, miRNA-TF co-regulation network analyses have helped investigators identify important regulatory motifs and understand cellular regulatory mechanisms in several diseases [19]. In gene regulatory networks, typical FFL motifs are composed of three nodes: miRNA, TF, and their jointly regulated target gene. Recently, FFL-based mixed regulatory networks have served as promising tools to elucidate complex diseases, such as schizophrenia [20], glioblastoma multiforme [21], ovarian cancer [22], non-small cell lung cancer [23], colorectal cancer [19], and osteosarcoma [24].

In this study, we employed a regulatory network-based approach for systematically investigating gene regulation patterns in CL. Using the CL candidate genes that we systematically collected and curated, we first examined four types of regulatory pairs: miRNA-gene, miRNA-TF, TF-gene and TF-miRNA. Based on these interaction pairs, we obtained three types of FFLs and named them motifs A, B, and C. Then, we used these motifs to construct four networks (motif A network, motif B network, motif $C$ network, and combined network of motifs $A, B$, and $\mathrm{C}$ ). We further evaluated the functional features of genes in these networks using the pathway enrichment analysis based on the Gene Ontology (GO) and the Kyoto Encyclopedia of Genes and Genomes database (KEGG) pathway annotations. We further used an independent gene expression data set (GEO accession number: GSE7759) to verify the findings from the combined network. This dataset could verify $64.8 \%$ of TF-gene edges. We extracted ten hub genes and identified three modules from the combined network for functional enrichment analysis. Notably, by investigating the network modules, we found the signaling pathway that regulate pluripotency of stem cells was significantly involved in $\mathrm{CL}$; such finding has not been reported before. Our network analysis also highlighted a CL subnetwork that is related to Wnt pathway, and pinpointed five miRNAs, hsa-mir-374b, hsa-mir-381, hsa-mir-374, hsa-mir-27b and $h s a-m i r-497$, involved in it. Through a gene expression and network topological analysis, we identified two 
of these miRNAs, hsa-mir-27b and hsa-mir-497, were essential for the activation of the Wnt pathway. Previous studies reported that both of these miRNAs were involved in the etiology of CL. Thus, our miRNA-TF-mediated regulatory network analysis was consistent with previous studies, suggesting the efficacy of the approach. Taken together, our integrated miRNA-TF mediated regulatory networks are useful for detecting genes, regulators and networks, which provides insights into the regulation of CL. Future experimental studies of these top candidates and regulations will help advance our knowledge in CL biology and the CL etiology.

\section{Results}

Integrative framework for identification of critical diseasecausing genes based on networks

Figure 1 elaborates our combinatory framework to construct comprehensive miRNA-TF co-regulatory networks and sort out critical genes and modules in CL. These networks were composed of FFL regulations among three main components: miRNAs, TFs and genes. In this study, we used the genes and miRNAs that we searched from several literature databases followed by careful, manual curation for human CL (Additional file 1). Specifically, through an extensive exploration of three major biomedical databases, Medline ${ }^{\bullet}$ (National Library of Medicine), NCBI's PubMed, and Embase ${ }^{\circ}$ (Excerpta Medica Database, Elsevier), we compiled a list of 162 genes with linkage or mutations reported in human individuals with $\mathrm{CL}$, and regarded them as candidate genes for human CL (Fig. 1a). We also identified 16 miRNAs that targeted these 162 genes from four miRNA-target interaction databases: miRanda, PITA, TargetScan and miRTarBase [25-28] (Additional file 1). In these networks, there are four types of regulatory interactions: TF regulation of gene expression (TF-gene), TF regulation of miRNA expression (TF-miRNA), miRNA regulation of gene expression (miRNA-gene) and miRNA regulation of TF expression (miRNA-TF). (Fig. 1b). To incorporate these regulations into miRNA-TF co-regulatory networks, we included only the miRNA- and TF-mediated FFLs (Fig. 1c and d). We combined different types of FFLs into networks and then performed functional analysis. To evaluate whether the computational analysis-driven interplays between regulators and their targets were consistent with the previous studies, we introduced a network edge validation strategy using an independent expression profiling data set (GEO accession number: GSE7759). We pinpointed important hub nodes (TFs, miRNAs, and genes) from a combined network and performed Gene Ontology (GO) gene set enrichment analysis of their targets (Fig. 1e). The GO enrichment results were consistent with previous studies and these hubs could be ideal candidates for further biological experiments. We extracted three network modules from the combined network and found the enrichment of genes involved in "the signaling pathway regulating pluripotency of stem cells" (Fig. 1f). Genes related to the Wnt pathway, a well-known pathway in the etiology of CL, were extracted and used to construct a network, in which we identified candidate diseasing-causing miRNAs (Fig. 1g). For the gene list derived from our procedures, we further performed GO and KEGG pathway enrichment analysis (Fig. 1h).

Highly confident regulatory relationships among miRNAs, TFs and genes

Table 1 summarizes four types of potential regulatory relationships (TF-gene, TF-miRNA, miRNA-gene, and miRNA-TF) and measurement methods we used in this study.

Prediction of miRNA targets has often a high false positive rate. To reduce such noises, we used the miRNA-target interactions in humans that were predicted by at least two of the four datasets: TargetScan, miRanda, PITA and miRTarBase [25-28] using stringent criteria. We obtained 77 miRNA-gene pairs and 21 miRNA-TF pairs. To obtain the regulatory relationships for each TF with its genes or miRNAs, we investigated TFs and their binding motifs in the TRANSFAC Professional database and predicted TF-gene and TF-miRNA interactions by using the $\mathrm{Match}^{\mathrm{TM}}$ program [29]. To minimize false positive results, we selected only the regulatory relationships conserved well among human, rat, and mouse [20, 23]. These data processes resulted in 671 TF-gene pairs and 76 TF-miRNA pairs (Table 1; Additional file 2).

\section{CL-specific miRNA-TF mediated regulatory networks with 64.8\% TF-gene edges being verified}

Our networks were established based on the transcriptional regulation of TFs (e.g. TF-gene, TF-miRNA) tightly coupled with the post-transcriptional regulation of miRNAs (e.g. miRNA-gene, miRNA-TF). These two types of gene regulators tend to form 3-node FFLs (each FFL has a TF, a miRNA and a joint target gene). In this study, we only considered three types of 3-node FFL motifs in CL. We named them motifs A, B, and C (Table 2). Based on the four types of regulatory relationships obtained above, a total of 128 FFLs belonged to these three types of FFL motifs (Table 2; Additional file 3).

In motif $\mathrm{A}$, the $\mathrm{TF}$ regulates its targeted protein-coding gene (non-TF gene) and miRNA at the transcriptional level, and the miRNA regulates its targeted protein-coding gene (non-TF) at the post-transcriptional level. In motif $\mathrm{B}$, the TF regulates its targeted protein-coding gene (non-TF gene) at the 


\section{Construction of $\mathrm{CL}$-specific miRNA-TF regulatory network}

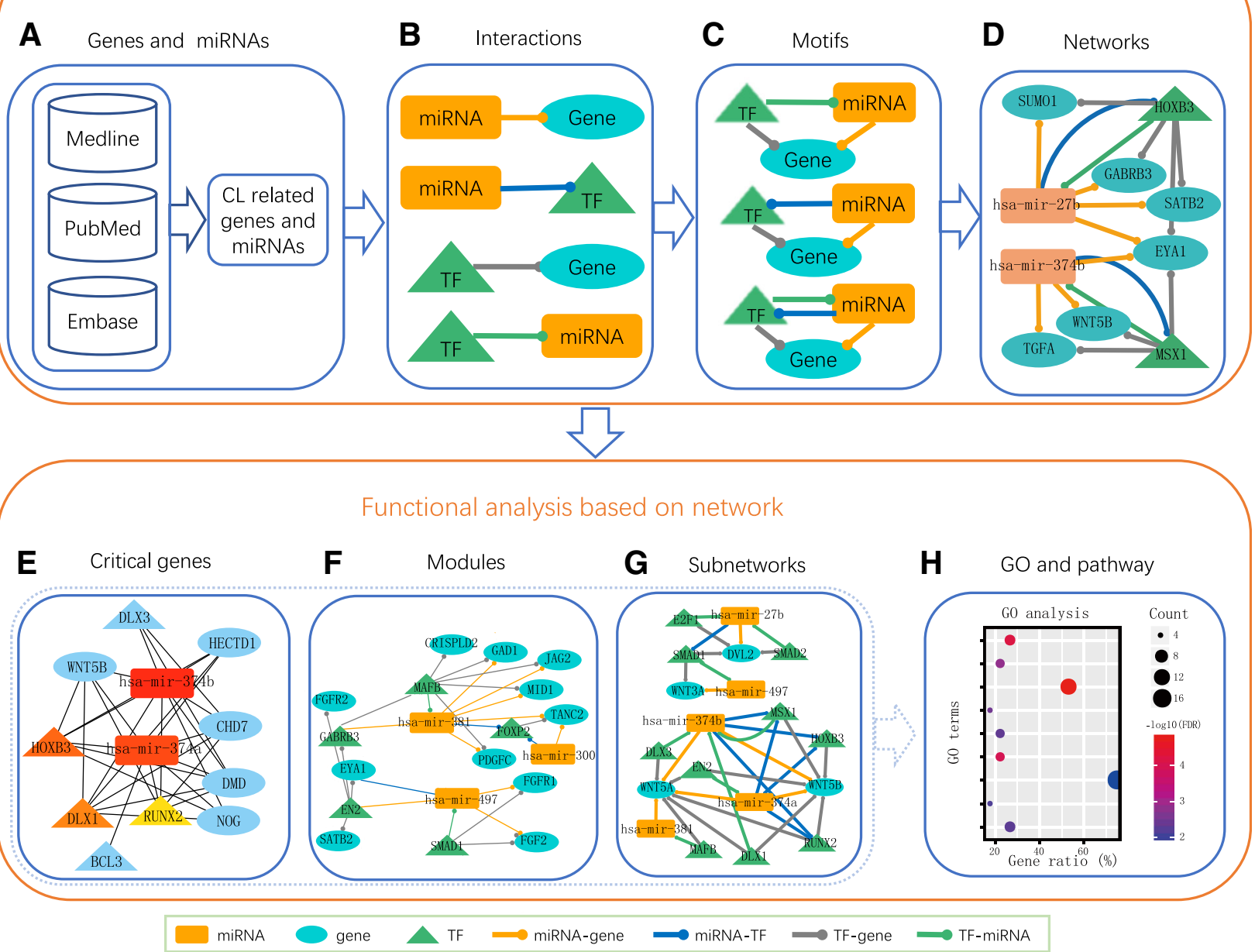

Fig. 1 Schematic flowchart for constructing and analyzing the comprehensive miRNA-TF co-regulatory networks in cleft lip (CL). (a) CL-related genes were collected and manually curated from main biomedical databases including Medline, PubMed and Embase. The miRNAs targeting these genes were identified from four miRNA-target interaction databases: miRanda, PITA, TargetScan and miRTarBase. (b) Four types of regulation relationships: TF-gene, TF-miRNA, miRNA-TF and miRNA-gene. (c) Three regulatory motifs measured by feed forward loop (FFL). (d) Construction of the miRNA-TF co-regulatory network. The combined network of all FFLs consisted of 8 miRNAs, 15 TFs, 26 genes and 163 edges. (e) Identification of critical genes (TFs, miRNAs and genes) and analysis of their potential functions. (f) Identification of network modules and analysis of their potential functions. (g) Extracting miRNA-TF co-regulatory subnetworks based on a specific pathway (Wnt pathway). (h) GO and pathway enrichment analysis. Blue ellipse: gene; orange rectangle: miRNA; green triangle: TF; blue line: miRNA-TF; orange line: miRNA-gene; green line: TF-miRNA; gray line: TF-gene

Table 1 Summary of the integrated regulatory relationships among miRNAs, TFs and genes

\begin{tabular}{|c|c|c|c|c|c|}
\hline Relationship & Number of pairs & Number of miRNAs & Number of genes & Number of TFs & Method $^{a}$ \\
\hline miRNA-gene & 77 & 9 & 37 & / & $A$ \\
\hline miRNA-TF & 21 & 8 & / & 12 & $A$ \\
\hline TF-gene & 671 & / & 126 & 27 & B \\
\hline TF-miRNA & 76 & 17 & / & 22 & B \\
\hline Total & 845 & 18 & 127 & 27 & \\
\hline
\end{tabular}

${ }^{a}$ In the Method column, (A) regulation relationships were supported by at least two of these databases: TargetScan, miRanda, PITA and miRTarBase; (B) TRANSFAC Match $^{T M}$ method was used for identification of TF-target relationship 
Table 2 Summary of 3-node feed forward loops (FFLs)

\begin{tabular}{|c|c|c|c|c|c|c|c|c|}
\hline \multirow[b]{2}{*}{ Motif } & \multirow[b]{2}{*}{ \#FFLs } & \multicolumn{3}{|c|}{ Number of nodes } & \multicolumn{4}{|c|}{ Number of pairs } \\
\hline & & TFs & miRNAs & Genes & miRNA-gene & miRNA-TF & TF-gene & TF-miRNA \\
\hline A & 71 & 12 & 7 & 26 & 39 & / & 68 & 17 \\
\hline B & 50 & 7 & 6 & 16 & 30 & 13 & 31 & / \\
\hline C & 7 & 2 & 2 & 6 & 7 & 2 & 7 & 2 \\
\hline Total & 128 & 16 & 12 & 28 & 69 & 13 & 99 & 19 \\
\hline
\end{tabular}

transcriptional level, and the miRNA regulates both TF and protein-coding gene (non-TF gene) at the post-transcriptional level. In motif $\mathrm{C}$, the regulators (TF and miRNA) mutually regulate each other, while the TF regulates the targeted protein-coding gene (non-TF gene) at the transcriptional level, and the miRNA regulates the targeted protein-coding gene (non-TF gene) at the post-transcriptional level. We constructed motif A, $\mathrm{B}$, and $\mathrm{C}$ co-regulatory networks by merging these motif $\mathrm{A}, \mathrm{B}$, and C FFLs, respectively (Fig. 2a, b, and c). In addition, we merged all these motif FFLs to construct a combined regulatory network (Fig. 2d). The combined network consisted of 8 miRNAs, 15 TFs, 26 genes and 163 edges (Additional file 4).

To examine whether the regulatory relationships between TFs and targeted genes were reproducible, we used an independent gene expression data set (GEO accession number: GSE7759). To determine if an edge (the link between two nodes in the network) could be verified, we required that the co-expression relationship between TF and gene should be observed in the validation data set, and we used the Pearson correlation coefficient $|r|>0.3$ to nominate the co-expression. Furthermore, the Pearson correlation $P$-values were required to be less than 0.05 with its false discovery rate (FDR) being less than 0.1 . The detailed network edge validation approach was described in the Materials and methods section. Among the 88 TF-gene edges, 57 (64.8\%) edges were verified by the independent data set (Additional file 5).

Next, we investigated enrichment of GO and KEGG pathway annotations in these TFs and genes from each motif network. Our results were insightful into CL biological functions (Fig. 3; Additional file 6). There were 17, 9, 1 important GO terms and 13, 6, 0 key KEGG pathways in motif $\mathrm{A}, \mathrm{B}$, and $\mathrm{C}$ networks, respectively. Among them, "negative regulation of canonical Wnt signaling pathway (GO:0090090)", "palate development (GO:0060021)", "signaling pathways regulating pluripotency of stem cell (hsa04550)", "Hippo signaling pathway (hsa04390)", "Wnt signaling pathway (hsa04310)" have been previously reported in CL [30-35]. Interestingly, as CL occurs with cleft palate at approximately $50 \%$ of all craniofacial cleft cases, "palate development (GO:0060021)" was observed across all the three motif networks (Additional file 6).

\section{Prominent hub genes in regulatory networks}

Based on maximal clique centrality (MCC), a novel topological analysis method introduced in [36], we pinpointed hub nodes in the combined network using the cytoHubba plugin (version 0.1) for Cytoscape (version 3.6) [36]. We obtained ten hub nodes, including three miRNAs, five TFs and one protein-coding gene (non-TF gene) (Fig. 4a).

For each hub TF and each miRNA, we first extracted its targets and then performed GO analysis for them using the DAVID software (version 6.8) [37]. We observed a set of four hub TFs and three hub miRNAs whose targets were significantly enriched in $11 \mathrm{GO}$ terms that are closely related to CL-specific biological processes (hypergeometric test $P<0.05$, followed by the Benjamini-Hochberg procedure for multiple testing correction [38]) (Fig. 4b; Additional file 7). The results from functional enrichment of target genes implied that these hub regulators might have essential roles in CL development. Targets of four TFs (DLX1, EN2, HOXB3, and MAFB) were enriched in the process of positive regulation of cell division that is an important biological process in the lip development. Prior to completion of formation of the upper lips, the lateral nasal process has a climax of cell division that leave upper lips susceptible to teratogenic insults, and any perturbation in growth at this critical moment can result in malfunction of the closure mechanism [2, 39]. Furthermore, targets of three TFs (DLX1, EN2, and HOXB3) were significantly associated with the following biological processes: wound healing and positive regulation of transcription from RNA polymerase II promoter. These biological processes have previously reported to be associated with CL [40-42].

Network modules revealed novel pathways involved in $\mathrm{CL}$ The Markov clustering (MCL) algorithm [43] is a highly efficient and reliable network clustering algorithm based on simulating random walks within an interaction network. We used MCL in order to divide regulatory network into gene interaction modules. With the inflation value set to 2.0, MCL detected three modules from the combined network (Fig. 5a). Each module was composed of a set of miRNAs, TFs, and genes that were topologically adjacent. These modules were taken for further functional analysis. 


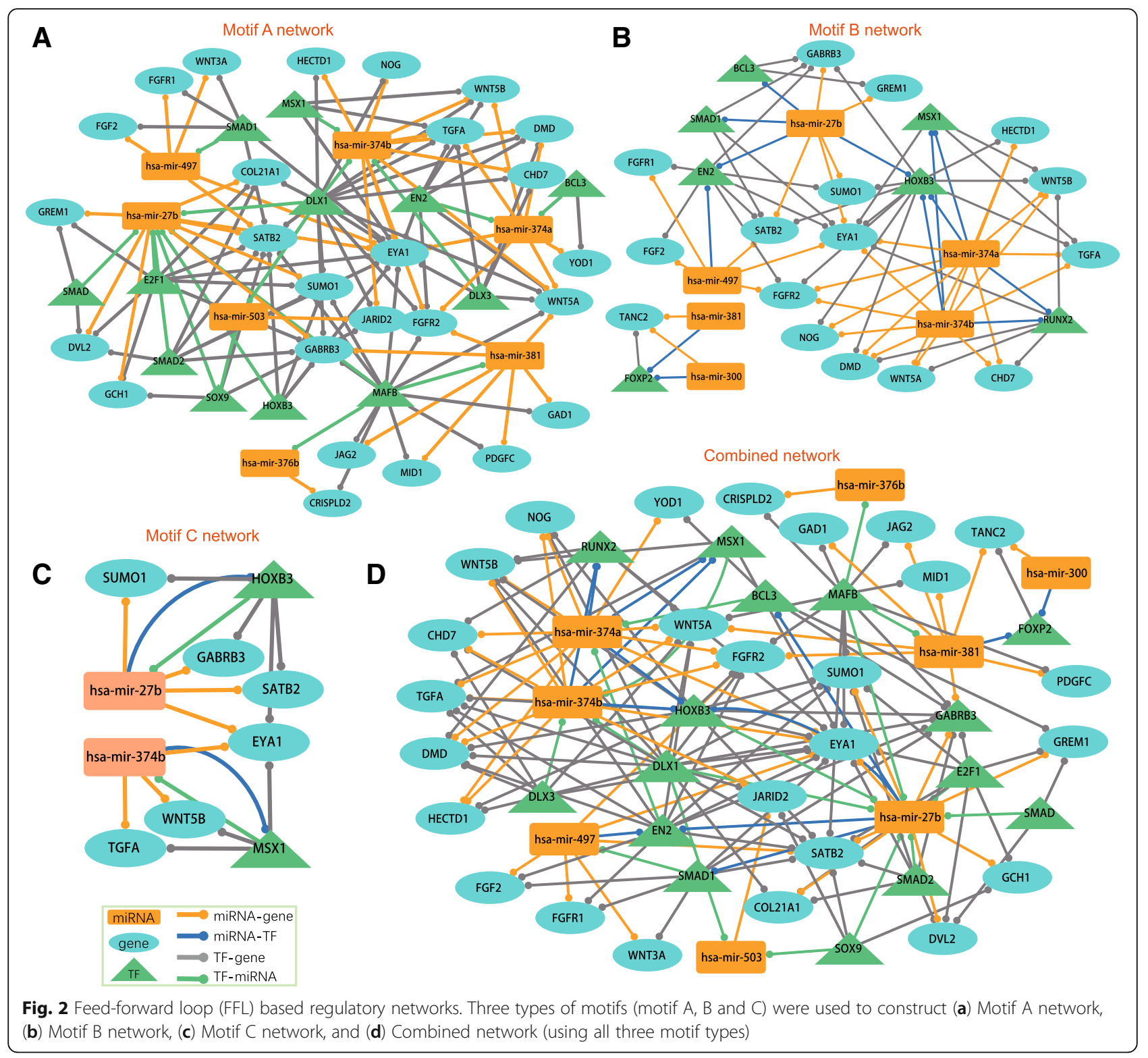

We aimed to reveal critical regulations that might control the etiology of CL. Pathway analysis has been demonstrated as a helpful method to investigate the biological mechanisms implicated in the pathogenesis [44]. We explored enriched pathways in these regulatory modules. Modules 1 and 3 were enriched in several pathways (FDR $<0.05$, hypergeometric test followed by Benjamini-Hochberg multiple testing correction), while module 2 was not enriched in any pathways (Additional file 8; Fig. 5a and b). This suggests that through identification of modules we can narrow down candidate genes and thus help us find critical regulations. Interestingly, both modules 1 and 3 were enriched in the signaling pathway regulating the pluripotency of stem cells. Although this pathway has not been reported to be involved in CL yet, we could find some evidence in this pathway. SOX2 is known as one of the core genes in pluripotency and one of the transcriptional factors crucial for the reprogramming of mature cells into pluripotent stem cells $[45,46]$. SOX2 is highly correlated with FOXE1 [47], whose congenital mutations cause cleft palate and hypothyroidism [48].

\section{Identification of potential disease-causing miRNAs in Wnt pathway in $\mathrm{CL}$}

Several studies have demonstrated that Wnt pathway is involved in non-syndromic CL [30, 49, 50]. For example, mutations in the WNT3 gene dominate autosomal recessive tetra-amelia with CL $[1,32]$. A single SNP (dbSNP ID: rs7205289) located in pre-miR-140 contributed to 
A

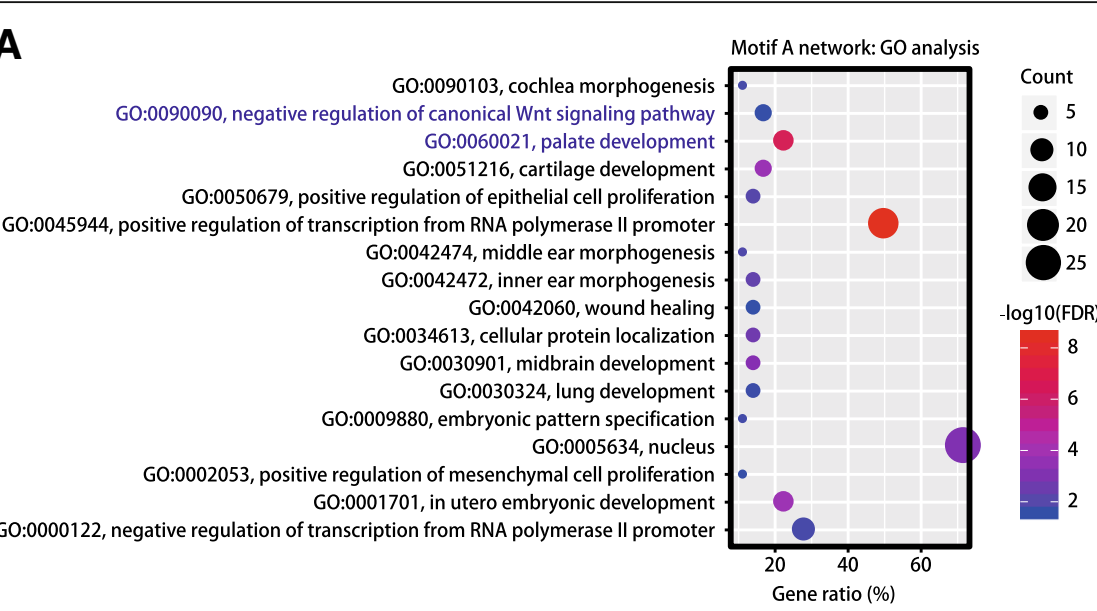

B

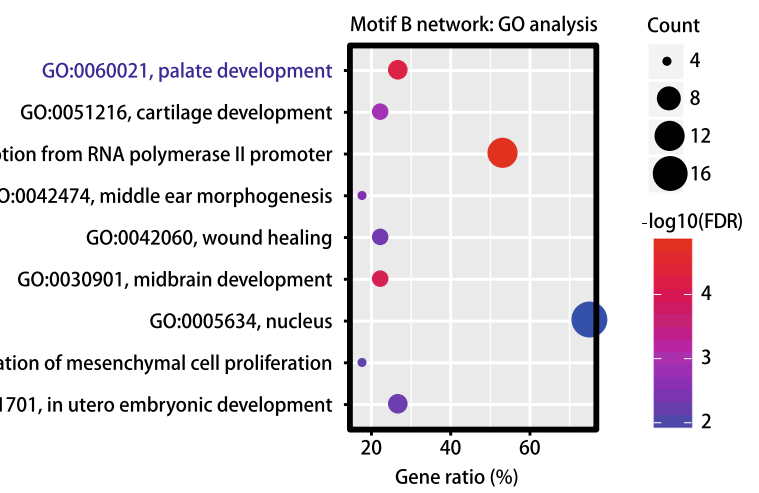

C

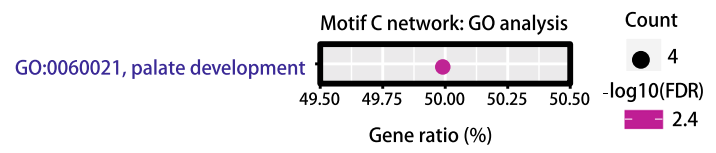

D

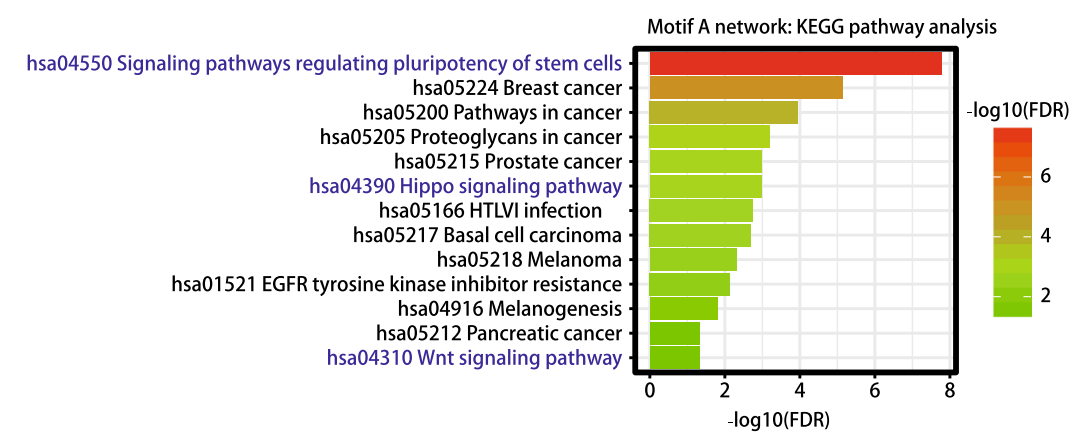

$\mathbf{E}$

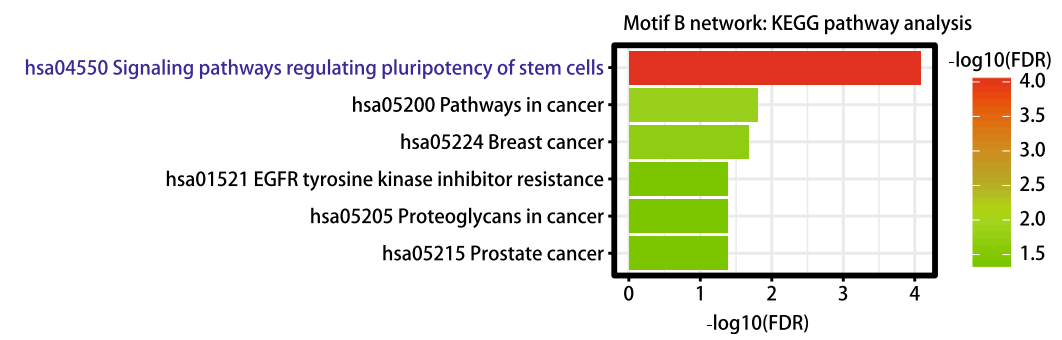

Fig. 3 Enrichment of GO and KEGG pathways in TFs and genes in each motif network. (a) GO analysis of TFs and genes in motif A network. (b) GO analysis of TFs and genes in motif B network. (c) GO analysis of TFs and genes in motif C network. (d) KEGG pathway enrichment analysis of TFs and genes in motif A network. (e) KEGG pathway enrichment analysis of TFs and genes in motif B network. FDR: false discovery rate 


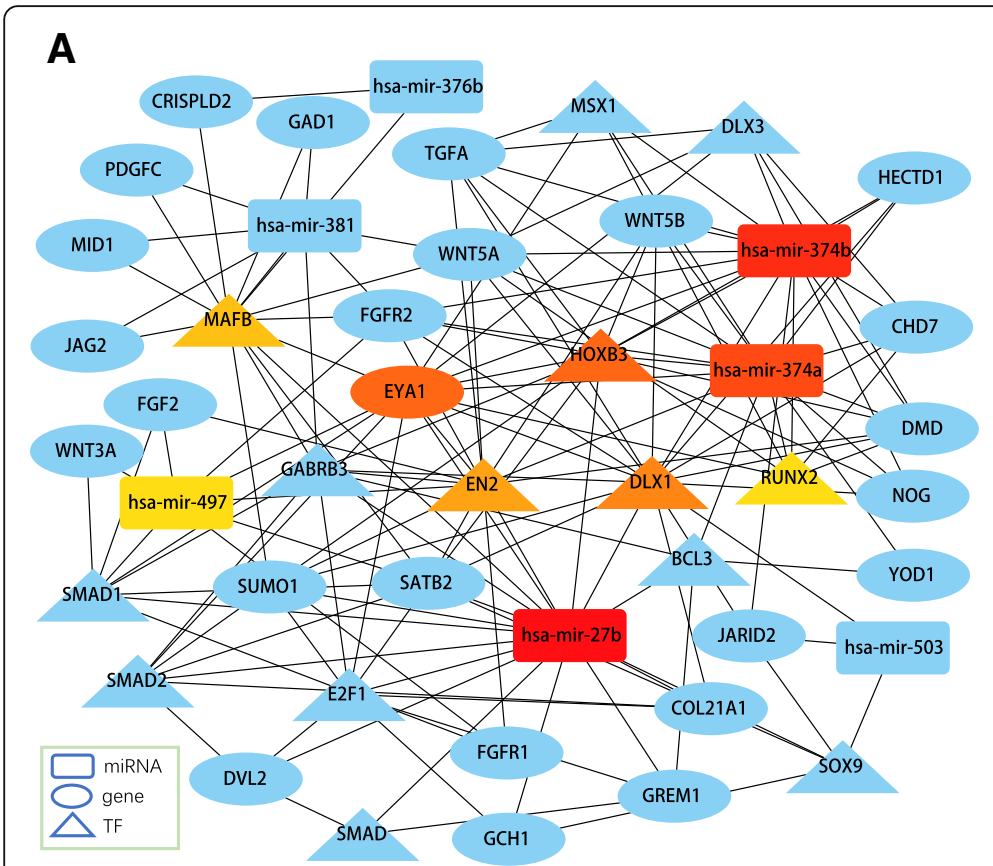

B

Fig. $4 \mathrm{Hub}$ nodes in the combined network and GO enrichment analysis of their targets. (a) Hub nodes in the combined network. Hub nodes are indicated with a color scheme from highly essential (red) to essential (yellow). (b) Enriched GO terms of the targeted genes of the hub miRNAs and TFs in (a)

the susceptibility of non-syndromic cleft palate (NSCP) by influencing the processing of $m i R-140$ [51]. These findings have motivated us to further analyze miRNAs in the Wnt pathway as candidate causative regulators for CL.

Based on the KEGG pathway database [52], our miRNA-TF co-regulatory networks could recruit four genes in the Wnt pathway: three WNT family genes (WNT3A, WNT5A, and WNT5B) and the DVL2 gene. We extracted two subnetworks from the miRNA-TF co-regulatory combined network by merging all the motifs that included at least one of these four genes. These subnetworks included 36 edges, five miRNAs, ten TFs and four genes (Fig. 6a and b). The subnetworks showed some interesting results. For example, WNT3A and WNT5A that appeared in these subnetworks were well-known involved in regulating upper lip fusion and mid-face development and were therefore especially strong candidates for an etiological role in CL [53, 54]. Furthermore, mutations in $M S X 1$ were associated with tooth agenesis and orofacial clefting in human [55].

Subnetwork 2 was composed of the core Wnt pathway gene WNT3A and five regulators (two miRNAs: hsa-mir-27b and hsa-mir-497; and three TF genes: SMAD1, $S M A D 2$, and E2F1). Among these five regulators, we identified two miRNAs (hsa-mir-27b and hsa-mir-497) as promising $\mathrm{CL}$ critical hub nodes. The direction of the network edges demonstrated that WNT3A expression could be regulated by two miRNAs at the post-transcriptional level and regulated by $S M A D 1$ at the transcriptional level (Fig. 6b). Of note, SMAD1-DVL2 and SMAD1-WNT3A were strongly validated by the independent validation data set (Fig. 6c). WNT3A expression could occur through up- or down-regulation of miRNAs that target WNT3A.

Previous studies have shown that hsa-mir-27b is upregulated in the $\mathrm{CL}$ region compared to normal tissues $[16,56]$. Another previous study has confirmed the downregulation of hsa-mir-497 in fibroblasts from individuals with non-syndromic isolated cleft palate [57]. In the independent validation data set, $S M A D 1$ and WNT3A were downregulated and upregulated compared to normal tissues, respectively. Although there has been no report on $h s a-m i r-27 b$ as a CL-causing miRNA, our analysis could lead to the following putative regulation pathways (Fig. 6c): hsa-mir-27b inhibits $S M A D 1$ expression and then SMAD1 inhibits WNT3A expression. Collectively, hsa-mir-27b and hsa-mir-497 may be candidate CL miRNAs involved in the etiology of CL via the Wnt pathway.

\section{Discussion}

During the past decade, association studies (e.g. GWAS) and candidate gene studies have identified a good number of genes or loci related to CL. The identification of candidate $\mathrm{CL}$ genes has traditionally been based on developmental analyses and gene expression [1]. However, with very scarce human samples available for CL gene 


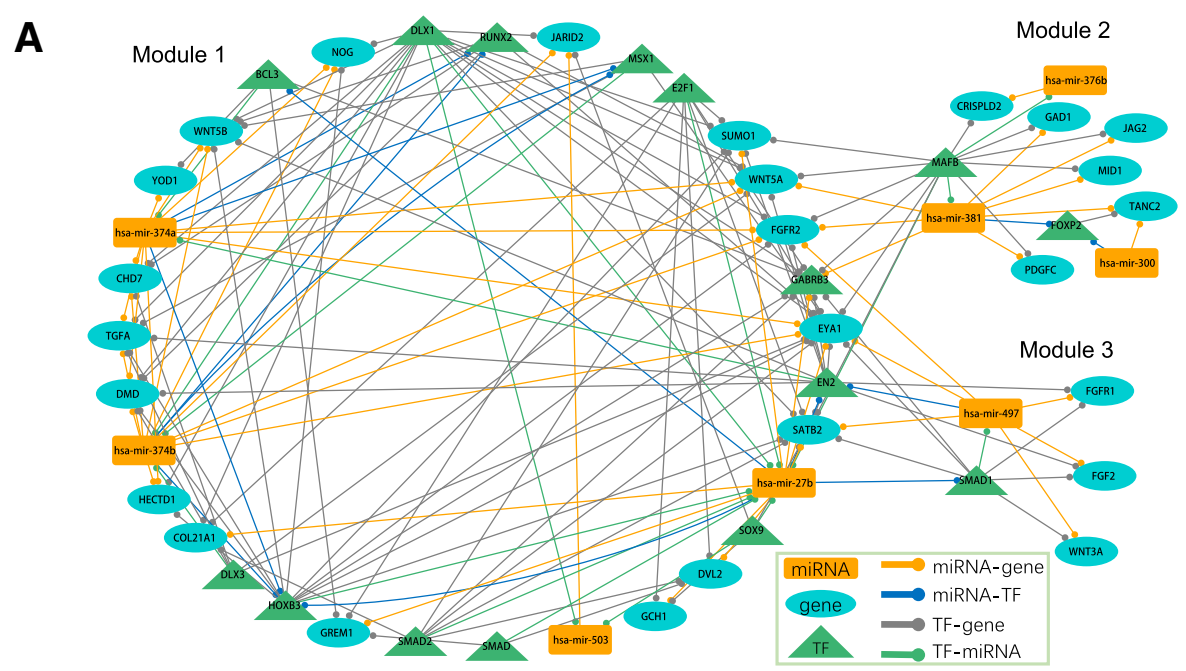

B Module 1 pathway analysis hsa04550 Signaling pathways regulating pluripotency of stem cells hsa05200 Pathways in cancer hsa05166 HTLV-I infection hsa04390 Hippo signaling pathway hsa05212 Pancreatic cancer hsa05224 Breast cancer hsa05217 Basal cell carcinoma

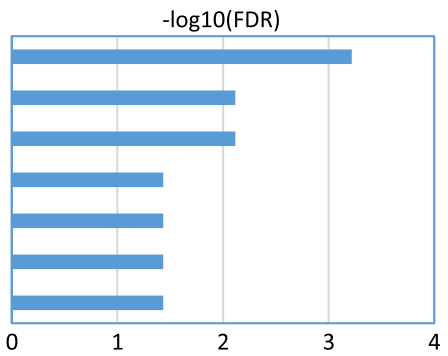

C Module 3 pathway analysis hsa05224 Breast cancer hsa05205 Proteoglycans in cancer hsa05200 Pathways in cancer hsa05218 Melanoma

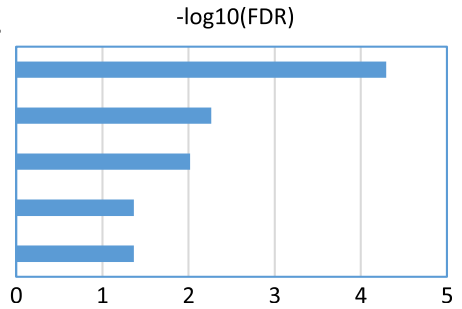

Fig. 5 Network modules and their functional analysis. (a) Network modules identified from the combined network. (b) KEGG pathway analysis of TFs and genes in module 1. (c) KEGG pathway analysis of TFs and genes in module 3

expression assay, such studies have mostly been performed in model animals, particularly in mice and zebrafish. The assumption is that the function and phenotypic changes related to the CL genes and mutagenesis in animals can be similarly reflected in humans. With this scenario, the mutations that are associated with $\mathrm{CL}$ in human studies (e.g. GWAS) may be better understood through manipulation in animals or measured in early craniofacial development. Since lip development involves dynamic changes and regulation, gene regulation network based on FFLs would be a promising approach to explore the molecular regulation among those previously identified as CL candidate genes. In principle, gene regulation network assumes that many of the disease-causing genes are prone to collaboratively take effect at the network level or biological pathway rather than at an individual gene level [58].
In this study, we constructed TF and miRNA co-regulatory networks using our curated CL genes in human, aiming to find insightful regulatory motifs and networks that are related to the etiology of CL. Our framework started with the curated genes and miRNAs in human CL; this effort of candidate gene selection and curation has not been done in the dental biology field yet. We inferred confident regulatory relationships among the TFs, miRNAs and genes using a whole set of computational tools and algorithms. Based on these relationships, we focused on 3-node FFL motifs to build up CL-specific regulatory networks. This TF-miRNA-gene 3-node FFL is a power way to examine the gene regulation at both the transcription and post-transcriptional levels. However, one critical issue in such a computational approach is how to best control the false positives during the establishment of the regulatory interaction 


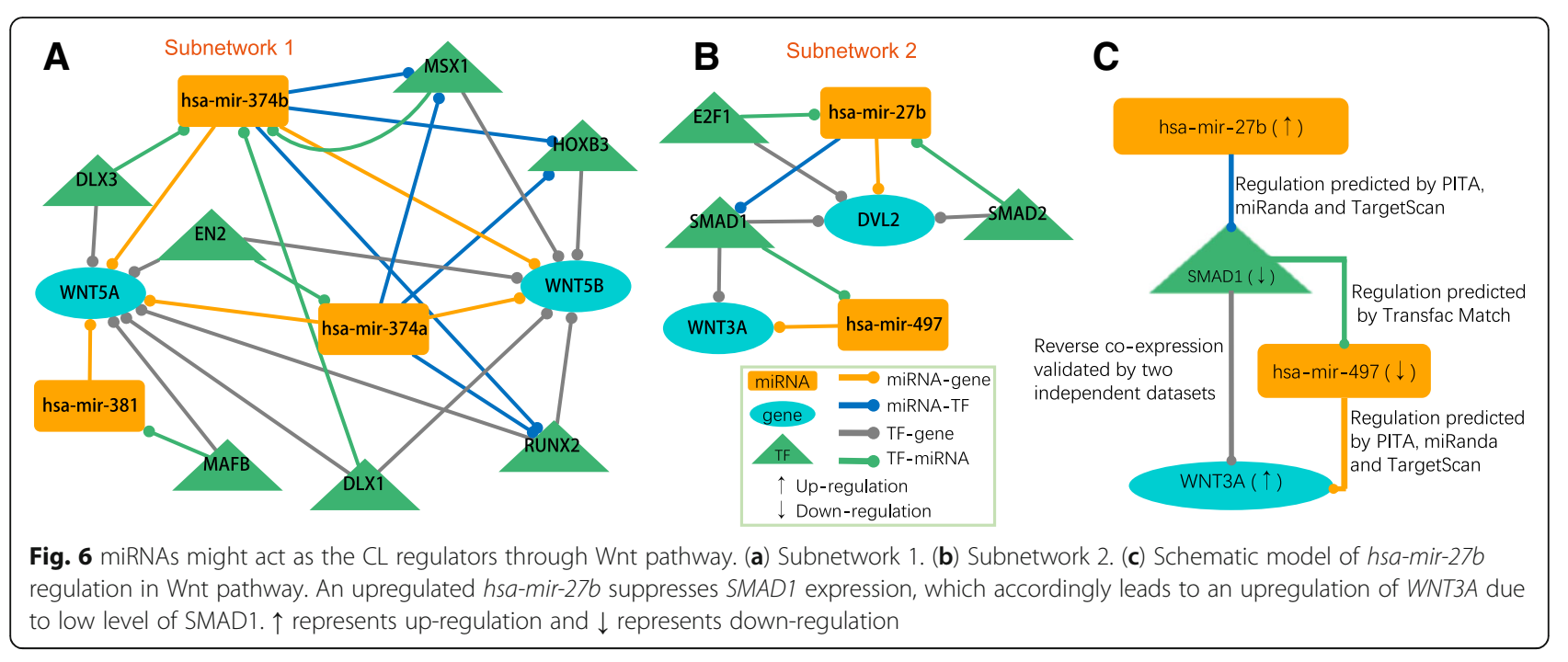

relationships. To reduce the rate of false-positive outcomes, we took the following steps. First, we selected the most recognized software, algorithms and annotated databases to predict the regulation pairs between TF-gene, TF-miRNA, miRNA-gene, and miRNA-TF. Second, we applied stringent parameters to choose miRNA-target and TF-target relationships (see Materials and methods). Third, we required the predicted pairs from more than one databases or tools. Thus, the results seemed reliable, as we verified by using an independent dataset. Overall, our framework can be applied to other complex diseases to detect regulatory relationships, important genes, critical molecular modules, and disease-causing regulators.

Among the results, the three hub miRNAs (hsa-mir-27b, hsa-mir-374a, and hsa-mir-497) and four hub TF genes (DLX1, EN2, HOXB3, and MAFB) were identified with relatively higher number of targets. The target genes of these regulators were involved in the CL-related biological processes. Therefore, these hub nodes may be vital candidates for further functional validation. In addition, through the analysis of network modules, we found the enrichment of genes involved in "the signaling pathway regulating pluripotency of stem cells". Although no previous studies have revealed this important pathway in CL yet, there was indirect evidence to support the underlying association between this pathway and CL.

Another important contribution of this study is the CL-specific miRNA-TF regulatory network. To our knowledge, there is no such a regulatory network analysis in the dental biology field. To identify potential disease-causing regulators, we segmented the combined regulatory network and separated it into relatively small but functionally crucial subnetworks for specific pathways that have been previously reported in CL. We then used independent gene expression data sets and performed network topology analyses in order to identify critical miRNAs in these small subnetworks. Specifically, we took Wnt pathway as a case and found two critical miRNAs (Fig. 6c). Among them, hsa-mir-27b has already been reported in independent studies to be up-regulated in CL $[16,56]$. Another study has shown that hsa-mir-497 was down-regulated in CL [56]. The prior evidence that supports our network results demonstrated the effectiveness of our regulatory approach. The observation in Fig. 6 may reflect a more complicated regulation scheme in cellular system, and such scenario will not be detected by a typical canonical pathway or signaling pathway approach. Different from traditional pathway analysis, which usually focuses on protein-coding genes, our approach integrates miRNAs, TFs and genes into special regulatory pathways. Such pathways might be useful for explanation of mechanism of diseases.

There are four limitations in our study. First, to identify regulatory relationships among TFs, miRNAs and genes, we used stringent criteria in order to reduce false positives. This strategy might exclude some true positive regulations. Second, we did not determine the repression or activation relationship in each interaction pair (e.g. TF activates or represses a gene). Third, these networks do not bear scale-free topology in a typical biological network because the pairs of regulation used for network construction are more likely related. Fourth, to our best knowledge, there were no published samples which had matched miRNA and mRNA expression available for construction of large-scale regulatory networks. The FaceBase project [59] has generated both mRNA and miRNA expression data in mice, but has not included both miRNA and mRNA from the same tissue or sample yet. Such data will likely be available in the near future since genome technologies are under rapid advancement, 
and we will expand our analysis when such data are released.

\section{Conclusions}

In this study, we have first identified TF-gene and miRNA-gene using our manually curated CL genes and other regulation databases. Then, we constructed comprehensive miRNA-TF mediated co-regulatory networks specific for human CL. Within these networks, we identified critical hub miRNAs, TFs and genes, which might take important roles in regulation process of CL. In addition, we found several novel pathways possibly associated with etiology of CL when we compared the results of functional analysis of different network modules. We also demonstrated that the CL-specific regulatory networks had critical disease-causing miRNAs. Moreover, we constructed a subnetwork containing human CL-related genes involved in Wnt pathway. Through network topological and functional analyses of Wnt pathway subnetwork, two critical miRNAs have been identified with the support from previous studies. This study not only unveils novel miRNAs for further experimental design but also provides further insight into regulatory mechanisms of human CL. To our knowledge, this is the first TF-miRNA mediated regulatory network in dental disease.

\section{Methods}

Human $\mathrm{CL}$ genes and related miRNAs

Genes involved in the pathology of CL were collected from three databases: Medline (Ovid), PubMed (NLM), and Embase (Ovid). After manual curation (every related paper was manually read), we obtained a list of 162 genes with mutations or association reported in individuals with $\mathrm{CL}$, and considered them as candidate genes for human CL. Our bioinformatics analysis suggested that 16 miRNAs might be post-transcriptional regulators of CL genes. (Details in another study in house; Additional file 1).

\section{TF-mediated gene/miRNA regulation}

The promoter sequences $(-1000 /+200 \mathrm{bp}$ of the transcription start site (TSS)) of human protein-coding genes and precursor miRNAs were obtained from the UCSC Table Browser (https://genome.ucsc.edu) [60] as described by Sun et al. [21]. The search of the TF binding sites was conducted using the Match ${ }^{\text {tw }}$ program [61], which was available in the TRANSFAC ${ }^{\circ}$ Professional version (release 2016.1) [29]. A high-quality TRANSFAC - matrix library and pre-calculated cutoffs were used to minimize false positive matches. To constrain the search, TFs containing a matrix score of $>0.95$ and a core score of 1.00 were selected.
miRNA-mediated gene/TF regulation

The candidate targeted genes of miRNAs were analyzed using bioinformatics analysis with multiple target prediction algorithms, including TargetScan (version 7.1), miRanda (August 2010 Release), PITA (version 6) and miRTarBase (Release 7.0) [25-28]. To obtain reliable miRNA-target pairs, a predicted miRNA-target pair for further analysis was supported by at least two of these public canonical miRNA-target databases.

\section{Validation of TF-gene regulation relationships}

The mouse model is well-established to facilitate studies of the mechanism of human craniofacial development [62]. This is because mouse and human have strikingly similar craniofacial development and well conserved molecular mechanism [62-64]. To validate the TF-gene regulatory interactions in the combined network, an independent mouse gene expression data set was obtained from the Gene Expression Omnibus (GEO) [65]: accession number GSE7759 [66]. GSE7759 contains 35 samples from the developing maxillary processes that eventually form the upper lip. Their gene levels were measured using Affymetrix Mouse Genome 4302.0 Array (Affymetrix, USA). Microarray data set was normalized with Robust Multi-Chip Averaging (RMA) [67]. TF-gene pairs with the Pearson correlation coefficient (PCC) being $>0.3$ or $<-0.3$ and $P$-value less than 0.05 (FDR less than 0.1) were selected (Additional file 5).

\section{Network and subnetwork generation, analyses, and functional evaluation}

In this work, we constructed three major networks. The first network was the CL-specific miRNA-TF mediated gene regulatory network, which was generated by converging all 3-node motifs (Fig. 2d). To determine the hubs in this network, cytoHubba, a java plugin for Cytoscape software, was employed. CytoHubba provides eleven topological analysis methods. We used Maximal Clique Centrality (MCC), since MCC has a better performance on the precision of predicting essential components from network among the eleven methods.

The second one consisted of three modules generated by the MCL (Markov Clustering) algorithm [43] (Fig. 5a). The MCL algorithm is designed specifically for clustering of simple or weighted graphs. The MCL algorithm finds cluster structure in graphs by a mathematical bootstrapping procedure. The results of MCL depend on the choice of an inflation parameter (I). We applied MCL to the networks constructed with default parameters (inflation parameter = 2.0) to identify the functional clusters. Clusters with less than three nodes were removed as less biological meaning.

The third one was the subnetwork for Wnt pathway (Fig. 6a and b). By reviewing the KEGG database (http:// www.genome.jp/kegg/), we found WNT3A, WNT5A, 
WNT5B and DVL2 were involved in Wnt pathway. We extracted all the motifs which contained at least one of these four genes to construct a subnetwork, and then inferred the potential function of miRNAs in Wnt pathway.

WebGestalt [68] was used to examine the KEGG pathways that were enriched with a gene list. The significance level of pathways was set to FDR (Benjamini-Hochberg) less than 0.05. The DAVID Functional Annotation Tool was used to identify enriched Gene Ontology (GO) annotations [37].

Statistical analyses were conducted using R 3.3.1 (the R Foundation for Statistical Computing; https://www.r-project.org/). The networks were visualized using the Cytoscape software version 3.6 (http://cytoscape.org/) [69].

\section{Additional files}

\section{Additional file 1: Table S1. Human CL related 162 genes and 16 miRNAs. (XLSX $11 \mathrm{~kb})$ \\ Additional file 2: Table S2. Regulatory relationships among miRNAs,} TFs and genes. (XLSX $22 \mathrm{~kb}$ )

Additional file 3: Table S3. 3-node feed forward loops (FFLs). (XLSX 12 $\mathrm{kb})$

Additional file 4: Table S4. Regulatory relationships among miRNAs, TFs and genes in the combined network. (XLSX $13 \mathrm{~kb}$ )

Additional file 5: Table S5. Validation of TF-gene pairs using an independent gene expression data set. (XLSX $18 \mathrm{~kb}$ )

Additional file 6: Table S6. Enrichment of GO and KEGG pathway annotations of TFs and genes in each motif network. (XLSX $86 \mathrm{~kb}$ )

Additional file 7: Table S7. GO analysis of targets of hub miRNAs and TFs. (XLSX $12 \mathrm{~kb}$ )

Additional file 8: Table S8. KEGG pathway analysis of network modules. (XLSX $11 \mathrm{~kb}$ )

\section{Abbreviations}

3-UTR: 3' untranslated region; BH: the Benjamini-Hochberg procedure; CL: cleft lip; CP: cleft palate; DAVID: the database for annotation, visualization and integrated discovery; FBL: feedback loop; FDR: the false discovery rate; FFL: feed-forward loop; GO: Gene Ontology; GWA: genome wide association; KEGG: the Kyoto Encyclopedia of Genes and Genomes database; MCL: Markov clustering; miRNA: microRNA; mRNA: messenger RNA; NSCL/ P: non-syndromic cleft lip with or without cleft palate; NSCP: nonsyndromic cleft palate; PCC: Pearson correlation coefficient; SNP: single nucleotide polymorphism; TF: transcription factor; TFBS: transcription factor binding site; TGFA: transforming growth factor, alpha; TSS: transcriptional start site; UCSC: the University of California Santa Cruz

\section{Acknowledgements}

We would like to thank the members of Bioinformatics and Systems Medicine Laboratory (BSML) for valuable discussion.

\section{Funding}

This work was partially supported by National Institutes of Health grant [R01LM012806 (to ZZ), R01DE026767 (to Jl), R03DE026208 (to Jl), R03DE026509 (to Jl), R03 DE027393 (to ZZ), R03DE028103 (to ZZ), and R03DE027711 (to Jia)], Natural Science Basic Research Plan in Shaanxi Province of China (2017JM6024 to AL), Natural Science Foundation of Shaanxi Provincial Department of Education (17JK0572 to AL), Teaching Research Foundation of Xi'an University of Technology (xjy1866 to AL). Publication charges for this article have been funded by authors' academic fund. The funders had no role in the study design, data collection and analysis, decision to publish, or preparation of the manuscript.

\section{Availability of data and materials}

All the data used in this study are from public sources cited in our reference list. Also, additional files, which may be needed to reproduce the results presented in the manuscript, are made available as additional files.

\section{About this supplement}

This article has been published as part of BMC Medical Genomics Volume 12 Supplement 1, 2019: Selected articles from the International Conference on Intelligent Biology and Medicine (ICIBM) 2018: medical genomics. The full contents of the supplement are available online at https:// bmcmedgenomics.biomedcentral.com/articles/supplements/volume-12supplement-1.

\section{Authors' contributions}

$\mathrm{ZZ}, \mathrm{AL}$, and PJ designed the project, AL, AS, MG, JI, PJ, and ZZ collected the data, $A L$ and $G Q$ analyzed the data, AL drafted the manuscript, and $Z Z$, J, and PJ edited the manuscript. All authors approved the final manuscript.

Ethics approval and consent to participate

Not applicable.

Consent for publication

Not applicable.

\section{Competing interests}

The authors declare that they have no competing interests.

\section{Publisher's Note}

Springer Nature remains neutral with regard to jurisdictional claims in published maps and institutional affiliations.

\section{Author details}

'Shaanxi Key Laboratory for Network Computing and Security Technology, School of Computer Science and Engineering, Xi'an University of Technology, Xi'an 710048, Shaanxi, China. ${ }^{2}$ Center for Precision Health, School of Biomedical Informatics, The University of Texas Health Science Center at Houston, 7000 Fannin St., Suite 820, Houston, TX 77030, USA. ${ }^{3}$ School of Software, Xidian University, Xi'an 710071, Shaanxi, China. ${ }^{4}$ Department of Diagnostic and Biomedical Sciences, School of Dentistry, The University of Texas Health Science Center at Houston, Houston, TX 77054 USA. ${ }^{5}$ Center for Craniofacial Research, The University of Texas Health Science Center at Houston, Houston, TX 77054, USA. ${ }^{6}$ MD Anderson Cancer Center UTHealth Graduate School of Biomedical Sciences, Houston, TX 77030, USA

\section{Published: 31 January 2019}

\section{References}

1. Dixon MJ, Marazita ML, Beaty TH, Murray JC. Cleft lip and palate: understanding genetic and environmental influences. Nat Rev Genet. 2011; 12(3):167-78.

2. Mossey PA, Little J, Munger RG, Dixon MJ, Shaw WC. Cleft lip and palate Lancet. 2009:374(9703):1773-85.

3. Xu M, Ma L, Lou S, Du Y, Yin X, Zhang C, Fan L, Wang H, Wang Z, Zhang W. Genetic variants of microRNA processing genes and risk of non-syndromic orofacial clefts. Oral Dis. 2018;24(3):422-8.

4. Kobayashi GS, Alvizi L, Sunaga DY, Francis-West P, Kuta A, Almada BVP, Ferreira SG, de Andrade-Lima LC, Bueno DF, Raposo-Amaral CE. Susceptibility to DNA damage as a molecular mechanism for nonsyndromic cleft lip and palate. PLoS One. 2013;8(6):e65677.

5. Yu Y, Zuo X, He M, Gao J, Fu Y, Qin C, Meng L, Wang W, Song Y, Cheng Y. Genome-wide analyses of non-syndromic cleft lip with palate identify 14 novel loci and genetic heterogeneity. Nat Commun. 2017;8:14364

6. Cai Y, Patterson KE, Reinier F, Keesecker SE, Blue E, Bamshad M, Haddad J. Copy number changes identified using whole exome sequencing in nonsyndromic cleft lip and palate in a Honduran population. Birth defects research. 2017;109(16):1257-67. 
7. Alvizi L, Ke X, Brito LA, Seselgyte R, Moore GE, Stanier P, Passos-Bueno MR. Differential methylation is associated with non-syndromic cleft lip and palate and contributes to penetrance effects. Sci Rep. 2017;7(1):2441.

8. Ardinger $\mathrm{H}$, Buetow K, Bell Gl, Bardach J, VanDemark D, Murray J. Association of genetic variation of the transforming growth factor-alpha gene with cleft lip and palate. Am J Hum Genet. 1989;45(3):348.

9. Lu Y, Liu Q, Xu W, Li Z, Jiang M, Li X, Zhao N, Liu W, Sui Y, Ma C. TGFA and IRF6 contribute to the risk of nonsyndromic cleft lip with or without cleft palate in Northeast China. PLoS One. 2013;8(8):e70754.

10. Gritli-Linde A. p63 and IRF6: brothers in arms against cleft palate. J Clin Invest. 2010;120(5):1386-9.

11. Thomason HA, Zhou H, Kouwenhoven EN, Dotto G-P, Restivo G, Nguyen BC, Little H, Dixon MJ, van Bokhoven H, Dixon J. Cooperation between the transcription factors p63 and IRF6 is essential to prevent cleft palate in mice. J Clin Invest. 2010;120(5):1561-9.

12. Moretti F, Marinari B, lacono NL, Botti E, Giunta A, Spallone G, Garaffo G, Vernersson-Lindahl E, Merlo G, Mills AA. A regulatory feedback loop involving p63 and IRF6 links the pathogenesis of 2 genetically different human ectodermal dysplasias. J Clin Invest. 2010;120(5):1570-7.

13. Ha M, Kim VN. Regulation of microRNA biogenesis. Nat Rev Mol Cell Biol. 2014;15(8):509.

14. He X, Yan Y-L, DeLaurier A, Postlethwait JH. Observation of miRNA gene expression in zebrafish embryos by in situ hybridization to microRNA primary transcripts. Zebrafish. 2011;8(1):1-8.

15. Nakamura $Y$, He X, Kato H, Wakitani S, Kobayashi T, Watanabe S, lida A, Tahara H, Warman ML, Watanapokasin R. Sox9 is upstream of microRNA-140 in cartilage. Appl Biochem Biotechnol. 2012;166(1):64-71.

16. Wang S, Sun C, Meng Y, Zhang B, Wang X, Su Y, Shi L, Zhao E. A pilot study: screening target miRNAs in tissue of nonsyndromic cleft lip with or without cleft palate. Experimental and Therapeutic Medicine. 2017; 13(5):2570-6.

17. Spitz F, Furlong EE. Transcription factors: from enhancer binding to developmental control. Nat Rev Genet. 2012;13(9):613.

18. Somel M, Guo S, Fu N, Yan Z, Hu HY, Xu Y, Yuan Y, Ning Z, Hu Y, Menzel C. MicroRNA, mRNA, and protein expression link development and aging in human and macaque brain. Genome Res. 2010;20(9):1207-18.

19. Wang H, Luo J, Liu C, Niu H, Wang J, Liu Q, Zhao Z, Xu H, Ding Y, Sun J, et al. Investigating MicroRNA and transcription factor co-regulatory networks in colorectal cancer. BMC bioinformatics. 2017;18(1):388.

20. Guo A-Y, Sun J, Jia P, Zhao Z. A novel microRNA and transcription factor mediated regulatory network in schizophrenia. BMC Syst Biol. 2010;4(1):10.

21. Sun J, Gong X, Purow B, Zhao Z. Uncovering microRNA and transcription factor mediated regulatory networks in glioblastoma. PLoS Comput Biol. 2012;8(7):e1002488.

22. Zhao M, Sun J, Zhao Z. Synergetic regulatory networks mediated by oncogene-driven microRNAs and transcription factors in serous ovarian cancer. Mol BioSyst. 2013;9(12):3187-98

23. Mitra R, Edmonds MD, Sun J, Zhao M, Yu H, Eischen CM, Zhao Z. Reproducible combinatorial regulatory networks elucidate novel oncogenic microRNAs in non-small cell lung cancer. Rna. 2014;20(9):1356-68.

24. Poos K, Smida J, Nathrath M, Maugg D, Baumhoer D, Korsching E. How microRNA and transcription factor co-regulatory networks affect osteosarcoma cell proliferation. PLoS Comput Biol. 2013;9(8):e1003210.

25. Chou C-H, Chang N-W, Shrestha S, Hsu S-D, Lin Y-L, Lee W-H, Yang C-D, Hong H-C, Wei T-Y. Tu S-J: miRTarBase 2016: updates to the experimentally validated miRNA-target interactions database. Nucleic Acids Res. 2015; 44(D1):D239-47.

26. John B, Enright AJ, Aravin A, Tuschl T, Sander C, Marks DS. Human microRNA targets. PLoS Biol. 2004;2(11):e363.

27. Kertesz M, lovino N, Unnerstall U, Gaul U, Segal E. The role of site accessibility in microRNA target recognition. Nat Genet. 2007;39(10):1278.

28. Lewis BP, Burge CB, Bartel DP. Conserved seed pairing, often flanked by adenosines, indicates that thousands of human genes are microRNA targets. Cell. 2005;120(1):15-20.

29. Matys V, Kel-Margoulis OV, Fricke E, Liebich I, Land S, Barre-Dirrie A, Reuter I, Chekmenev D, Krull M, Hornischer K. TRANSFAC ${ }^{\oplus}$ and its module TRANSCompel $^{\oplus}$ : transcriptional gene regulation in eukaryotes. Nucleic Acids Res. 2006:34(suppl_1):D108-10.

30. Rafighdoost $H$, Hashemi M, Asadi H, Bahari G. Association of single nucleotide polymorphisms in WNT genes with the risk of non-syndromic cleft lip with or without cleft palate. Congenital Anomalies. 2018; 58(4):130-5.
31. Vijayan V, Ummer R, Weber R, Silva R, Letra A. Association of WNT pathway genes with nonsyndromic cleft lip with or without cleft palate. The Cleft Palate-Craniofacial Journal. 2017;55(3):335-41.

32. Li C, Lan Y, Krumlauf R, Jiang R. Modulating Wnt signaling rescues palate morphogenesis in Pax9 mutant mice. J Dent Res. 2017;96(11):1273-81.

33. Bueno DF, Sunaga DY, Kobayashi GS, Aguena M, Raposo-Amaral CE, Masotti C, Cruz LA, Pearson PL, Passos-Bueno MR. Human stem cell cultures from cleft lip/palate patients show enrichment of transcripts involved in extracellular matrix modeling by comparison to controls. Stem Cell Rev Rep. 2011;7(2):446-57.

34. Illich DJ, Demir N, Stojković M, Scheer M, Rothamel D, Neugebauer J, Hescheler J, Zöller JE. Concise review: induced pluripotent stem cells and lineage reprogramming: prospects for bone regeneration. Stem Cells. 2011;29(4):555-63.

35. Li J, Zou J, Li Q, Chen L, Gao Y, Yan H, Zhou B, Li J. Assessment of differentially expressed plasma microRNAs in nonsyndromic cleft palate and nonsyndromic cleft lip with cleft palate. Oncotarget. 2016;7(52):86266.

36. Chin $\mathrm{C}-\mathrm{H}$, Chen S-H, Wu H-H, Ho C-W, Ko M-T, Lin C-Y. CytoHubba: identifying hub objects and sub-networks from complex interactome. BMC Syst Biol. 2014;8(4):S11.

37. Huang DW, Sherman BT, Lempicki RA. Systematic and integrative analysis of large gene lists using DAVID bioinformatics resources. Nat Protoc. 2008;4(1):44.

38. Benjamini $Y$, Hochberg Y. Controlling the false discovery rate: a practical and powerful approach to multiple testing. J R Stat Soc Ser B Methodol. 1995;57:289-300.

39. Blanton SH, Henry RR, Yuan Q, Mulliken JB, Stal S, Finnell RH, Hecht JT. Folate pathway and nonsyndromic cleft lip and palate. Birth Defects Research Part A: Clinical and Molecular Teratology. 2011;91(1):50-60.

40. Vicente-Dueñas C, González-Herrero I, García-Ramírez I, Sánchez-García I. MAFB (v-maf avian musculoaponeurotic fibrosarcoma oncogene homolog B). Atlas Genet Cytogenet Oncol Haematol. 2015;19(7):453-7.

41. Xu M, Xie A. Selection of key genes and regulators associated with Treacher Collins syndrome based on expression profiling analysis. Int J Clin Exp Med. 2017;10(5):8481-91.

42. Suzuki S, Marazita ML, Cooper ME, Miwa N, Hing A, Jugessur A, Natsume N, Shimozato K, Ohbayashi N, Suzuki Y. Mutations in BMP4 are associated with subepithelial, microform, and overt cleft lip. Am J Hum Genet. 2009;84(3): 406-11.

43. Enright AJ, Van Dongen S, Ouzounis CA. An efficient algorithm for largescale detection of protein families. Nucleic Acids Res. 2002;30(7):1575-84.

44. Wang L, Jia P, Wolfinger RD, Chen X, Grayson BL, Aune TM, Zhao Z. An efficient hierarchical generalized linear mixed model for pathway analysis of genome-wide association studies. Bioinformatics. 2011;27(5):686-92.

45. Takahashi K, Yamanaka S. Induction of pluripotent stem cells from mouse embryonic and adult fibroblast cultures by defined factors. Cell. 2006;126(4): 663-76.

46. Yu J, Vodyanik MA, Smuga-Otto K, Antosiewicz-Bourget J, Frane JL, Tian S, Nie J, Jonsdottir GA, Ruotti V, Stewart R. Induced pluripotent stem cell lines derived from human somatic cells. Science. 2007;318(5858):1917-20.

47. Bass AJ, Watanabe $H$, Mermel CH, Yu S, Perner S, Verhaak RG, Kim SY, Wardwell L, Tamayo P, Gat-Viks I. SOX2 is an amplified lineage-survival oncogene in lung and esophageal squamous cell carcinomas. Nat Genet. 2009;41(11):1238.

48. Clifton-Bligh RJ, Wentworth JM, Heinz P, Crisp MS, John R, Lazarus JH, Ludgate M, Chatterjee VK. Mutation of the gene encoding human TTF-2 associated with thyroid agenesis, cleft palate and choanal atresia. Nat Genet. 1998;19(4):399.

49. Yuan G, Singh G, Chen S, Perez KC, Wu Y, Liu B, Helms JA. Cleft palate and Aglossia result from perturbations in Wnt and hedgehog signaling. The Cleft Palate-Craniofacial Journal. 2017;54(3):269-80.

50. Karibozorg H F, Masoudian N, Saliminejad K, et al. Association of the WNT3 Variations and the risk of non-syndromic cleft lip and palate in a population of Iranian infants. Avicenna journal of medical biotechnology. 2018;10(3):168.

51. Li L, Meng T, Jia Z, Zhu G, Shi B. Single nucleotide polymorphism associated with nonsyndromic cleft palate influences the processing of miR-140. Am J Med Genet A. 2010;152(4):856-62.

52. Kanehisa M, Goto S, Sato Y, Furumichi M, Tanabe M. KEGG for integration and interpretation of large-scale molecular data sets. Nucleic Acids Res. 2011:40(D1):D109-14.

53. Chiquet BT, Blanton SH, Burt A, Ma D, Stal S, Mulliken JB, Hecht JT. Variation in WNT genes is associated with non-syndromic cleft lip with or without cleft palate. Hum Mol Genet. 2008;17(14):2212-8. 
54. Menezes R, Letra A, Kim AH, Küchler EC, Day A, Tannure PN, da Motta LG, Paiva K, Granjeiro JM, Vieira AR. Studies with Wnt genes and nonsyndromic cleft lip and palate. Birth Defects Research Part A: Clinical and Molecular Teratology. 2010;88(11):995-1000.

55. van den Boogaard M-JH, Dorland M, Beemer FA, van Amstel HKP. MSX1 mutation is associated with orofacial clefting and tooth agenesis in humans. Nat Genet. 2000;24(4):342.

56. Zou J, Li J, Li J, Ji C, Li Q, Guo X. Expression profile of plasma microRNAs in nonsyndromic cleft lip and their clinical significance as biomarkers. Biomed Pharmacother. 2016;82:459-66.

57. Schoen C, Glennon JC, Abghari S, Bloemen M, Aschrafi A, Carels CE, Von den Hoff JW. Differential microRNA expression in cultured palatal fibroblasts from infants with cleft palate and controls. Eur J Orthod. 2017:40(1):90-6.

58. Network CGAR. Comprehensive genomic characterization defines human glioblastoma genes and core pathways. Nature. 2008:455(7216):1061.

59. Brinkley JF, Fisher S, Harris MP, Holmes G, Hooper JE, Jabs EW, Jones KL, Kesselman C, Klein OD, Maas RL. The FaceBase consortium: a comprehensive resource for craniofacial researchers. Development. 2016; 143(14):2677-88

60. Casper J, Zweig AS, Villarreal C, Tyner C, Speir ML, Rosenbloom KR, Raney BJ, Lee CM, Lee BT, Karolchik D. The UCSC genome browser database: 2018 update. Nucleic Acids Res. 2017;46(D1):D762-9.

61. Kel AE, Gossling E, Reuter I, Cheremushkin E, Kel-Margoulis OV, Wingender E. MATCHTM: a tool for searching transcription factor binding sites in DNA sequences. Nucleic Acids Res. 2003;31(13):3576-9.

62. Nie X, Luukko K, Kettunen P. FGF signalling in craniofacial development and developmental disorders. Oral Dis. 2006;12(2):102-11.

63. Newbern J, Zhong J, Wickramasinghe RS, Li X, Wu Y, Samuels I, Cherosky N, Karlo JC, O'Loughlin B, Wikenheiser J. Mouse and human phenotypes indicate a critical conserved role for ERK2 signaling in neural crest development. Proc Natl Acad Sci. 2008;105(44):17115-20.

64. Lan Y, Ryan RC, Zhang Z, Bullard SA, Bush JO, Maltby KM, Lidral AC, Jiang R. Expression of Wnt9b and activation of canonical Wnt signaling during midfacial morphogenesis in mice. Dev Dyn. 2006;235(5):1448-54.

65. Edgar R, Domrachev M, Lash AE. Gene expression omnibus: NCBI gene expression and hybridization array data repository. Nucleic Acids Res. 2002; 30(1):207-10.

66. Feng W, Leach SM, Tipney H, Phang T, Geraci M, Spritz RA, Hunter LE, Williams T. Spatial and temporal analysis of gene expression during growth and fusion of the mouse facial prominences. PLoS One. 2009;4(12):e8066.

67. Bolstad BM, Irizarry RA, Åstrand M, Speed TP. A comparison of normalization methods for high density oligonucleotide array data based on variance and bias. Bioinformatics. 2003;19(2):185-93.

68. Wang J, Vasaikar S, Shi Z, Greer M, Zhang B. WebGestalt 2017: a more comprehensive, powerful, flexible and interactive gene set enrichment analysis toolkit. Nucleic Acids Res. 2017:45(W1):W130-7.

69. Saito R, Smoot ME, Ono K, Ruscheinski J, Wang P-L, Lotia S, Pico AR, Bader GD, Ideker T. A travel guide to Cytoscape plugins. Nat Methods. 2012;9(11): 1069.

Ready to submit your research? Choose BMC and benefit from:

- fast, convenient online submission

- thorough peer review by experienced researchers in your field

- rapid publication on acceptance

- support for research data, including large and complex data types

- gold Open Access which fosters wider collaboration and increased citations

- maximum visibility for your research: over $100 \mathrm{M}$ website views per year

At $\mathrm{BMC}$, research is always in progress.

Learn more biomedcentral.com/submissions 